\title{
Application of Alternate Group Explicit Method for Linear Boundary Value Problems
}

\author{
Sanjay $^{1}$, Yogesh khandelwal ${ }^{2}$, Rachana Khandelwal ${ }^{3}$ \\ Department of Mathematics $1,2,3$ \\ Jaipur National University, Rajasthan, India-302017
}

\begin{abstract}
In this paper, we show that by a certain change of variables any equation of a particular type can be transported in to a canonical form which is associated with its type. In this, we refine our method in such a manner that the solutions retain their order and accuracy even in the vicinity of the singularity. Further, to solve some boundary value problems, we use iterative method, that is, the alternating group explicit (AGE) method to solve linear equation.
\end{abstract}

Keywords: Boundary value problem, Principal part, Partial differential equation, AGE method, Newton AGE method

\section{Introduction}

A partial differential equation describes a relation between an unknown function and its partial derivatives. We discussed the family of second order linear equations for functions in two independent variables into three distinct types: hyperbolic, parabolic, and elliptic equations.

A partial differential equation is defines as

$F\left(x_{1}, x_{2}, \ldots \ldots, x_{n}, u_{,} u_{x_{1}, \ldots \ldots} u_{x_{n}} u_{x_{1} x_{1}}, u_{x_{1} x_{2}} \ldots \ldots \ldots, u_{x_{n} x_{n}} \ldots \ldots\right)=0$

where the unknown $u=u\left(x_{1}, x_{2}, \ldots \ldots \ldots x x_{n}\right)$ is a function of $\mathrm{n}$ variables and $u_{x_{j}, \ldots \ldots} u_{x_{1} x_{j}} \ldots \ldots$ are its partial derivatives.

Equation (1) is linear if $F$ is linear with respect to $u$ and all its derivatives, otherwise it is nonlinear.

In applied mathematics and theoretical physics three types of equations occur more commonly these are examples of linear equations $i_{*} e \cdot(a)$ Laplace's equation, (b) Wave equation, (c) Diffusion equation

The general equation of second-order linear differential equation for functions in two independent variables $x$ and $y$. Such an equation has the form

$F[u]=p u_{x x}+2 q u_{x y}+r u_{y y}+s u_{x}+t u_{y}+f u=g$

Here if $p, q, r$ are the functions of $x_{x} y, u_{v} u_{x}, u_{y}$ then equation (2) is called a quasi-linear $P D E$. where $p, q, r, s, t, f, g$ are given functions of $x$ and $y$ and $u(x, y)$ is the unknown function. We assume that the coefficients $p, q, r$ do not vanish simultaneously.

The operator

$F_{0}[u]=p u_{x x}+2 q u_{x y}+r u_{y y}$

that consists of the second(highest)order terms of the operator $F$ is called the principal part of $F$

\section{2: General form of partial differential equation}

A general form of $P D E$ where, $x \in R^{n}, u=u(x)$

$\sum_{i=1}^{n} \sum_{j=1}^{n} a_{i j} \frac{\partial^{2} u}{\partial x_{i} \partial x_{j}}+\sum_{i=1}^{n} b_{i} \frac{\partial u}{x_{i}}+b_{0}(x)=f(x)$

\section{3: Solution of Non-Linear Boundary Value Problems Using A Fourth Order Finite difference method:}

In Science and Engineering we have two point nonlinear singular boundary value problems occur frequently in many complex mathematical modeling problems. Such problems can pose considerable difficulties to achieve an analytic solutions and it is usually necessary to resort to numerical techniques. There are a variety of numerical strategies which can be applied i.e., finite difference, finite elements, splines, etc.

Finite difference formulation:

We consider boundary value problems of the form:

$L[u(x)] \equiv u^{s v}(x)+f\left(x, u, u^{s}\right)=0, \quad 0<x<1$

With boundary conditions: 
$u(0)=T, u(1)=S$

Where $T$ and $S$ are constants. We assume that for equation (1) to have an unique solution then $f\left(x, u, u^{l}\right)$ has continuous derivatives which satisfy

$$
\left|\frac{\partial f}{\partial w}\right| \leq Q^{*}, 0<M_{*} \leq \frac{\partial f}{\partial w} \leq M^{*}
$$

for some positive constants $Q^{*}, M$, and $M^{*}$. A uniform grid is now placed on the interval $[0,1]$. Let $a>0$ be the mesh spacing in the $x$-direction, so that $x t=t a, t=0(1) N+1$ with $(N+1) a=1$ and the solution of (1) is approximated by $u_{t}(t=1(1) N)$ which are the solution of the non-linear difference equation

$$
-\left(u_{t-1}-2 u_{t}-u_{t-1}\right)+\frac{\hbar^{2}}{12}\left[\bar{F}_{t+1}+\bar{F}_{t-1}+\bar{F}_{t+1}\right]=0, t=1(1) N
$$

with $u_{0}=T$ and $u_{\mathbb{N}+1}=S$. Note that, the accuracy of the equation (5) is of $O\left(a^{4}\right)$ for the difference solution of equation (3).

\section{The Age and Newton age Methods:}

\section{1 The Age Method:}

The tri-diagnol linear system of equations of order $N$ for the linear difference equation $a_{t} u_{t-1}+2 b_{t} u_{t}+c_{t} u_{t+1}=A B_{t}, t=1(1) N$

$$
T u=A B
$$

Where

$$
T=\left[\begin{array}{ccccc}
2 q_{1} & r_{1} & & & 0 \\
p_{2} & 2 q_{2} & r_{2} & & \\
\because & \ddots & \ddots & & \ddots \\
\cdots & p_{N-1} & 2 q_{N-1} & r_{N-1} \\
0 & & \ldots & & 2 q_{N}
\end{array}\right], \quad u=\left[\begin{array}{c}
u_{1} \\
u_{2} \\
\cdots \\
\cdots \\
u_{N}
\end{array}\right], A B=\left[\begin{array}{c}
A B_{1}-T_{1} u_{0} \\
A B_{2} \\
\cdots \\
\cdots \\
A B_{N}-r_{N} u_{N+1}
\end{array}\right]
$$

We consider the case of $N$ odd, the case of $N$ even follows easily.

We split the matrix $T$ into the sum of two matrices

$$
\mathrm{T}=\mathrm{P}_{1}+\mathrm{P}_{2}
$$

Where

$$
P_{1}=\left[\begin{array}{lllllll}
{\left[q_{1}\right.} & & & & & & \\
& {\left[\begin{array}{ll}
q_{2} & r_{2} \\
p_{a} & q_{3}
\end{array}\right]} & & & & \\
& & & \ddots & & & \\
& & & & & {\left[\begin{array}{cc}
q_{N-1} & r_{N-1} \\
p_{N} & q_{N}
\end{array}\right]}
\end{array}\right]_{N \times N}
$$

and

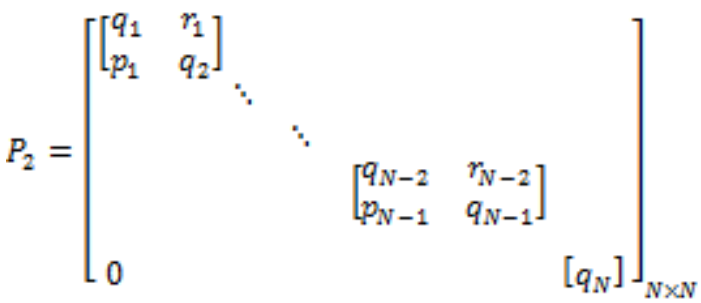

Since $P_{1}$ and $P_{2}$ are simply composed of $(2 \times 2)$ sub matrices and single diagonal entries, we can easily evaluate their eigen values. The eigen values $\emptyset$ of $P_{1}$ are given by $\emptyset=q_{1}$ and

$$
\left(\emptyset-q_{t}\right)\left(\varnothing-q_{t+1}\right)-p[t+1] r_{t}=0, \quad t=2(2) N-1
$$

Where, $p_{\mathrm{t}+1} r_{\mathrm{t}}>0$. The eigen values $\eta$ of $P_{2}$ are given by $\eta=q_{N}$ and

$$
\left(\eta-q_{t}\right)\left(\eta-q_{t+1}\right)-p_{t+1} r_{t}=0, t=1(2) N-2
$$

Assume that $\left(P_{1}+\mu_{1} I\right)$ and $\left(P_{2}+\mu_{2} I\right)$ are non singular matrices, then the alternating group explicit $(A G E)$ method is given by

$$
\begin{aligned}
\left(\mathrm{P}_{1}+\mu_{1} \mathrm{I}\right) \mathrm{u}^{(s+1 / 2)}= & \mathrm{AB}-\left(\mathrm{P}_{2}-\mu_{1} \mathrm{I}\right) \mathrm{u}^{(s)} \\
& \left(\mathrm{P}_{2}+\mu_{2} \mathrm{I}\right) \mathrm{u}^{(s+1)}=\mathrm{AB}-\left(\mathrm{P}_{1}-\mu_{2} \mathrm{I}\right) \mathrm{u}^{(s+1 / 2)}
\end{aligned}
$$

Where, $\mu_{1}$ and $\mu_{2}$ are the acceleration parameters of the first and second sweep of the $A G E$ method, respectively and $u^{(s+1 / 2)}$ is an intermediate vector. If we combine the equations (10a) and (10b), we get

Where,

$$
u^{g+1}=P u^{g}+J
$$




$$
\mathrm{P}=\left(\mathrm{P}_{2}+\mu_{2} \mathrm{I}\right)^{-1}\left(\mathrm{P}_{1}-\mu_{2} \mathrm{I}\right)\left(\mathrm{P}_{1}+\mu_{1} \mathrm{I}\right)^{-1}\left(\mathrm{P}_{2}-\mu_{1} \mathrm{I}\right)
$$

Is called the $A G E$ iteration matrix and

$$
\mathrm{J}=\left(\mathrm{P}_{2}+\mu_{2} \mathrm{I}\right)^{-1}\left(\mathrm{I}-\mathrm{P}_{1}-\mu_{2} \mathrm{I}\right)\left(\mathrm{P}_{1}+\mu_{1} \mathrm{I}\right)^{-1}
$$

Assume that $U$ is the exact solution of equation (7), then

$$
\left(P_{1}+P_{2}\right) U=A B
$$

And $\quad\left(P_{1}+\mu_{1} I\right) U=A B-\left(\mathrm{P}_{2}-\mu_{1} \mathrm{I}\right) U$

$$
\left(\mathrm{P}_{2}+\mu_{2} \mathrm{I}\right) U=A B-\left(\mathrm{P}_{1}-\mu_{2} \mathrm{I}\right) U
$$

Where $P$ is determine by equation (12).

$1^{\text {st }}$ iteration: Let $\varepsilon^{a}=u^{a}-U$ is the error vector associated with $u^{s}$ therefore from equation (5) and (10), we get $\left(P_{1}+\mu_{1} I\right) \varepsilon^{(s+1 / 2)}=-\left(\mathrm{P}_{2}-\mu_{1} \mathrm{I}\right) \varepsilon^{s}$

$\left(\mathrm{P}_{2}+\mu_{2} \mathrm{I}\right) \varepsilon^{s}=-\left(\mathrm{P}_{1}-\mu_{2} \mathrm{I}\right) \varepsilon^{(s+1 / 2)}$

and hence

$2^{\text {nd }}$ iteration, $\quad \varepsilon^{(s+1 / 2)}=P \varepsilon^{s}$

Where $P$ is given by equation (12).

For convergence it is required to prove that $\rho(P)<1$ for any $\mu_{1}>0$ and $\mu_{2}>0$.

Let

$P^{*}=\left(\mathrm{P}_{2}+\mu_{2} \mathrm{I}\right) \mathrm{P}\left(\mathrm{P}_{2}+\mu_{2} \mathrm{I}\right)^{-1}$

$=\left(\mathrm{P}_{2}-\mu_{2} \mathrm{I}\right)\left(P_{1}+\mu_{1} I\right)^{-1}\left(\mathrm{P}_{2}-\mu_{1} \mathrm{I}\right)\left(\mathrm{P}_{2}+\mu_{2} \mathrm{I}\right)^{-1}$

Then $P^{*}$ is similar to $P$, and hence $\rho(P)=\rho\left(P^{*}\right)$.

Now

$\|P\|_{2} \leq\left\|\left(\mathrm{P}_{1}-\mu_{2} \mathrm{I}\right)\left(P_{1}+\mu_{1} I\right)^{-1}\right\|_{2}\left\|\left(\mathrm{P}_{2}-\mu_{1} \mathrm{I}\right)\left(\mathrm{P}_{2}+\mu_{2} \mathrm{I}\right)^{-1}\right\|_{2}$

If $P_{1}$ has Eigen values $\emptyset_{1}, \emptyset_{2}, \emptyset_{g}, \ldots \ldots \ldots \ldots . \emptyset_{\mathbb{N}}$, then it is easy to verify that $R_{\varepsilon}\left(\emptyset_{\mathrm{i}}\right)>0$ and

$\left\|\left(\mathrm{P}_{1}-\mu_{2} \mathrm{I}\right)\left(P_{1}+\mu_{1} I\right)^{-1}\right\|_{2}=\rho\left\{\left(\mathrm{P}_{1}-\mu_{2} \mathrm{I}\right)\left(P_{1}+\mu_{1} l\right)^{-1}\right\}$

$$
=\max _{\emptyset_{\mathrm{t}}}\left|\frac{\phi_{\mathrm{p}_{\mathrm{t}}-\mu_{2}} \mid \mu_{1}}{\oint_{\mathrm{t}}}\right|<1
$$

Similarly,

$$
\left\|\left(\mathrm{P}_{2}-\mu_{1} \mathrm{I}\right)\left(\mathrm{P}_{2}+\mu_{2} \mathrm{I}\right)^{-1}\right\|_{2}<1
$$

Thus, from (17), we obtain, $\rho(P)=\rho\left(P^{*}\right) \leq\left\|P^{*}\right\|_{2}<1$.

Hence the convergence follows.

\section{2 The Newton Age Method:}

Now we discuss the Newton Age method for the non linear difference equation,

We define,

$$
u=\left[\begin{array}{c}
u_{1} \\
u_{2} \\
\vdots \\
\vdots \\
u_{N}
\end{array}\right], \quad \theta(u)=\left[\begin{array}{c}
\theta_{1}(u) \\
\theta_{2}(u) \\
\vdots \\
\vdots \\
\theta_{N}(u)
\end{array}\right]
$$

Now the Jacobian of $\theta(u)$ can be shown to be the $N$ th order tri-diagonal matrix

$$
R \equiv \frac{\partial \theta(u)}{\partial u}=\left[\begin{array}{ccccc}
2 q_{1}(u) & r_{1}(u) & & & \\
p_{2}(u) & 2 q_{2}(u) & r_{2}(u) & & \\
& \ddots & \ddots & & \ddots \\
& \ddots & \ddots & & \ddots \\
0 & & & p_{N}(u) & 2 q_{N}(u)
\end{array}\right]
$$

Where,

$$
\begin{array}{ll}
p_{t}(u)=\frac{\partial \theta_{t}}{\partial u_{t-1}}, & t=2(1) N \\
2 q_{t}(u)=\frac{\partial \theta_{t}}{\partial u_{t}}, & t=1(1) N \\
r_{t}(u)=\frac{\partial \theta_{t}}{\partial u_{t+1}}, & t=1(1) N-1
\end{array}
$$

Use $u_{0}=A$ and $u_{N+1}=B$ for computing $\theta_{t}(u), p_{t}(u), q_{t}(u), r_{t}(u)$.

We define

$$
u^{(s+1)}=u^{g}+\Delta u^{s} \quad s=0,1,2,3, \ldots \ldots \ldots
$$


Where $\Delta u^{a}$ is the solution of the system

$$
R \Delta u^{a}=-\theta\left(u^{s}\right) \quad s=0,1,2,3 \ldots \mathrm{mm}
$$

Here we use the $A G E$ iterative algorithm within inner loop to solve above equation

Consider

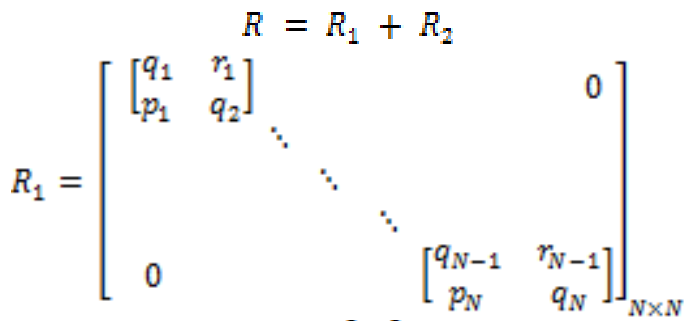

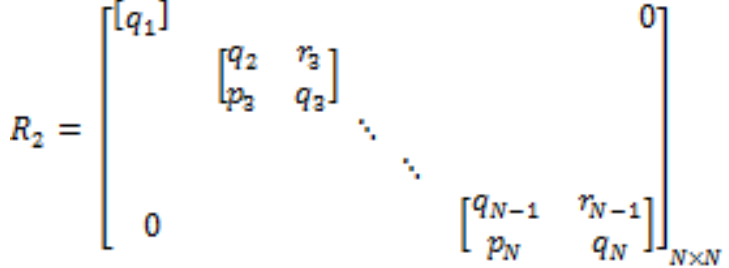

Where, we consider $N$ as even.

Then we can derive two steps alternating direction implicit methods as:

$\left(R_{1}+\mu_{1} I\right) \Delta u^{(s+1 / 2)}=-\theta\left(u^{g}\right)-\left(R_{2}-\mu_{1} I\right) \Delta u^{g}$

$\left(R_{2}+\mu_{2} I\right) \Delta u^{(s+1)}=-\theta\left(u^{s}\right)-\left(R_{1}-\mu_{2} I\right) \Delta u^{(s+1 / 2)}$

Since the matrices $\left(R_{1}+\mu_{1} I\right)$ and $\left(R_{2}+\mu_{2} I\right)$ consist of simple $(2 * 2)$ sub matrices then they can be easily inverted by inspection to give the Newton $A G E$ method.

$\Delta u^{(s+1 / 2)}=\left(R_{1}+\mu_{1} I\right)^{-1}-\left[\theta\left(u^{s}\right)-\left(R_{2}-\mu_{1} I\right) \Delta u^{(s+1)}\right.$

$\Delta u^{(g+1)}=\left(R_{2}+\mu_{2} I\right)^{-1}-\left[\theta\left(u^{\Omega}\right)-\left(R_{1}-\mu_{2} I\right) \Delta u^{(s+1 / 2)}\right.$

Where,

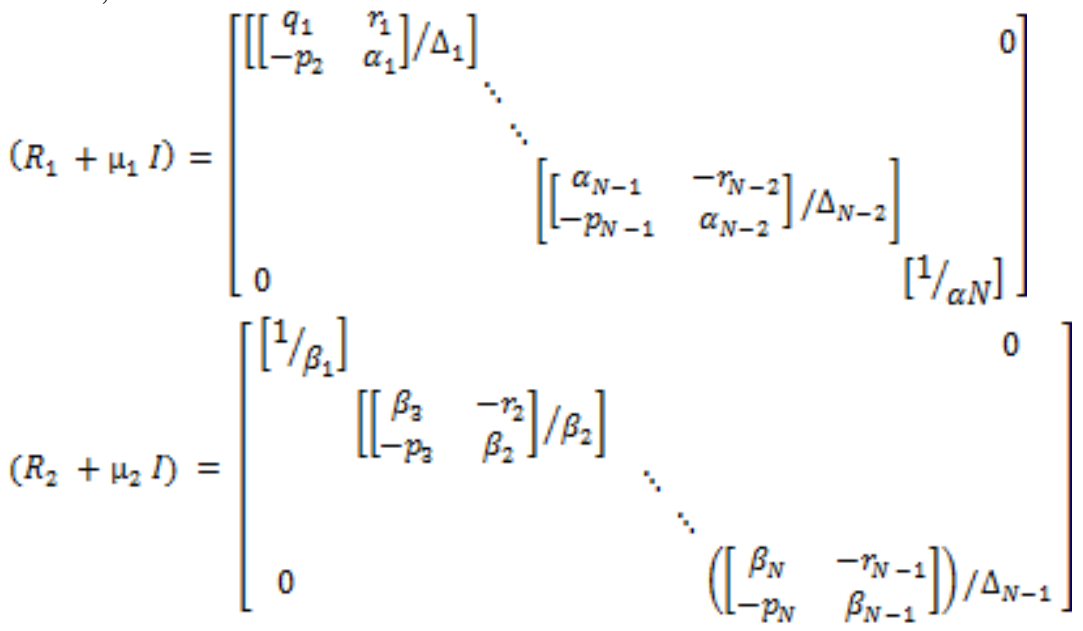

Where $\beta_{t}=q_{t}+\mu_{1}, t=1(1) N$ and $\Delta_{t}=\beta_{t} \beta_{t+1}-r_{t} p_{t+1}, \quad t=2(2) N-1$ and $\alpha_{t}=q_{t}+\mu_{2 x}$ $T=1(1) N$ and $\Delta_{t}=\alpha_{t} \alpha_{t+1}-r_{t} p_{t}+1, \quad t=1(2) N-2$.

Further the matrix product $\left(R_{1}+\mu_{1} I\right)^{-1}\left(R_{2}+\mu_{1} I\right)$ and $\left(R_{2}+\mu_{1} I\right)^{-1}\left(R_{1}-\mu_{2} I\right)$ can be simply determined and expressed as 3 terms recurrence relations in a manner suitable for parallel computing.

\section{Comparative results and Numerical Experiments:}

The following one singular problems are considered for computation whose exact solutions are known. We have compared the proposed $A G E$ iterative methods with the corresponding $S O R$ iterative method. We considered only 3-inner iterations.

Problem 5.1: Linear singular problem

$$
u^{s s}(x)+\frac{\mathscr{E}}{x^{b}}(x)=\beta x^{\beta-2}\left[\alpha+\beta-1+\beta x^{\beta}\right] u(x) \quad \text { Where, } \beta>0
$$

With the boundary conditions $y(0)=1, y(1)=e$ 
The exact solution is $(x)=e^{x^{g}}$. The root mean square (RMS) errors and the number of iterations both for AGE and SOR methods are tabulated in Table-1 for $\alpha=1_{s} \beta=1$.

Sol:

\begin{tabular}{|c|c|c|c|c|c|c|}
\hline & \multicolumn{4}{|c|}{$\alpha=1, \beta=1$} & \\
\hline & & \multicolumn{2}{|c|}{ SOR } & \multicolumn{2}{|c|}{$A G E$} & \\
\hline S.No & $N$ & $\omega$ & iter & $\omega$ & iter & RMS error \\
\hline 1 & 10 & 1.46 & 38 & 0.382 & 30 & $2.3758 e(-03)$ \\
\hline 2 & 20 & 1.500 & 75 & 0.290 & 65 & $1.8722 e(-04)$ \\
\hline 3 & 30 & 1.600 & 108 & 0.291 & 72 & $2.3725 e(-05)$ \\
\hline
\end{tabular}

Table-1

\section{Acknowledgements}

I hereby take the opportunity to thank everyone who helped me make this paper. my classmates Raj kumar, Vinay goyal ,Amit sharma, all had helped me make this paper and a great vote of thanks to all of them.

\section{References}

[1]. JAIN, M.K., IYENGAR, S.R.K. AND JAIN, R.K.,(1993) numerical methods for scientific and engineering computation, 3rded: wiley eastern ltd..

[2]. EVANS, D. J., Group Explicit Methods for the Numerical Solution of Partial Differential Equations, Gordon and Breach Science Publishers.

[3]. EVANS, D. J. And AHMAD, A.R.,(1996). Comparison of SOR and AGE Methods for Solution of the Two-Point Boundary Value Problem, Advances in Engineering Software.

[4]. VARGA, R. S., (1962). Matrix Iterative Analysis, Prentice Hall, Englewood Cliffs, New Jersey.

[5]. KELLER, H. B.,(1968). Numerical Methods for Two Point Boundary Value Problem, Blaisdell Pub. Co.

[6]. EVANS, D. J., (1985). Group Explicit Methods for Solving Large Linear Systems, Int. J. Computer Math., 17, 81-108.

[7]. WACHSPRESS, E. L.,(1962). Optimum Alternating Direction Implicit Iteration Parameters for a Model Problem, SIAM. J. Numerical. Analysis., $10,339-350$.

[8]. BENSON, A. The Numerical Solution of Partial Differential Equations by Finite Difference Methods, Ph.D. Thesis, Sheffield University.

[9]. COLLATZ, L.,(1960). Numerical treatment of Differential Equations, Springer, Berlin.

[10]. CHAWLA, M. M.,(1978). A Fourth Order Tri-diagonal Finite Difference Method for General Non-linear Two Point Boundary Value Problems with Mixed Boundary Conditions, J. Inst. Maths. Applics., 21, 83-93. 